\title{
Hallazgos de trombos intracardíacos por cardioresonancia no diagnosticados por ecocardiograma transtorácico.
}

\author{
Julián Noche ${ }^{1}$, Francisco Albornoz ${ }^{2}$, Rodrigo Gómez ${ }^{3}$, Brenda Flores ${ }^{3}$, Jorge Novoa ${ }^{4}$, Ricardo González ${ }^{4}$.
}

\begin{abstract}
(1) Jefe Programa Imagen Cardíaca Avanzada, Hospital Las Higueras de Talcahuano, Former Fellow Medicina Cardiovascular e Imagen Cardíaca Avanzada, Departamento de Medicina Cardiovascular, Vanderbilt University, Nashville, TN, USA. Docente programa de Post-Grado Cardiología, Facultad de Medicina, Universidad Andrés Bello, Chile.
\end{abstract}

(2) Jefe Medicina Cardiovascular Integrada UAT, Hospital Las Higueras de Talcahuano, Chile. Former Fellow Medicina Cardiovascular y Cardiología Intervencional, Vanderbilt University, Nashville, TN, USA. Master en Ciencias de la Investigación Clínica, Profesor Asociado de Medicina y Director de Programa de Postgrado de Cardiología, Facultad de Medicina, Universidad Andrés Bello.

(3) Cardiólogo Hospital Las Higueras de Talcahuano.

(4) Becado Cardiología, Postgrado de Cardiología, Hospital Higueras de Talcahuano, Facultad de Medicina, Universidad Andrés Bello

Facultad de Medicina Universidad Andrés Bello, Talcahuano, Chile. Hospital Las Higueras, Talcahuano, Chile. Vanderbilt University, Nashville, TN, USA.

Introducción: La Resonancia Magnética Cardíaca permite, entre otros, una superior localización espacial y caracterización tisular de las estructuras cardíacas, muchas veces omitidas por modalidades de alto uso como el ultrasonido.

Objetivo: Describir los hallazgos de trombos intracardíacos en una serie de pacientes enviados a estudio de resonancia cardíaca, en los cuales el ecocardiograma transtorácico no estableció el diagnóstico de trombo.

Método: Se realizó un análisis descriptivo de una serie de 62 casos, $72 \%$ hombres, entre 18 y 84 años, con disfunción sistólica severa $63 \%$; moderada $26 \%$ y normal $11 \%$. El $38 \%$ tenía historia presente o remota de Accidente Vascular Encefálico. Todos tenían ecocardiograma transtorácico sin trombos visibles y fueron enviados a resonancia cardíaca por estudio de cardiomiopatía no isquémica $41 \%$; viabilidad $41 \%$ y accidente vascular encefálico isquémico sin fuente embolígena demostrada $18 \%$ El estudio se efectuó en Vanderbilt University Medical Center, entre Julio del 2014 a Julio del 2015.

Resultados: Las imágenes, correspondientes a trombos cardíacos en los 62 casos, fueron localizados en ventrículo izquierdo en su gran mayoría (58 casos, $94 \%)$, orejuela de aurícula izquierda $(3,5 \%)$ y ambas orejuelas (1 caso, 1\%). Los trombos fueron en su gran mayoría laminares (52 casos, $84 \%$ ), sésiles (10 casos, $16 \%$ ) y ninguno pediculado.

Conclusión: La resonancia cardíaca es un método de imagen no invasivo muy superior a la ecocardiografía transtorácica en la identificación y localización de trombos intracardíacos, incluyendo pacientes con estudio de fuente embolígena negativos.

\footnotetext{
Correspondencia:

Dr. Julián Noche

Dirección: Alto Horno 777, Hospital Higueras de Talcahuano.

56-41-2687025.
}

ultranoche@hotmail.com 


\section{Intracardiac thrombus identified by Nuclear Magnetic Resonance not previously diagnosed by transthoracic echocardiography}

Cardiac Magnetic Resonance imaging allows, among others, a better localization and tissue characterization of cardiac structures, which may be missed by other imaging modalities, namely ultrasonography.

Aim: to describe the finding and characteristics of intracardiac thrombus in a series of patients referred for cardiac NMR imaging, whose previous transthoracic echocardiogram had been negative for the diagnosis of this condition.

Methods: the study is a description of NMR imaging findings in patients with intracardiac thrombus. There were 62 patients, $72 \%$ males, aged 18 to 64 years. LV systolic function was normal in $11 \%$, moderately decreased in $26 \%$ and severely depressed in $63 \%$ of patients. A remote or a recent cerebrovascular accident (CVA) was present in $38 \%$ of cases. All patients had a previous transthoracic echocardiogram with no evidence of thrombus. Patients had been referred for NMR with a diagnosis of non ischemic cardiomyopathy (41\%), study of myocardial viability (41\%) and CVA with unidentified embolic source $(18 \%)$. The study was performed at Vanderbilt University Medical Center, between July 2014 and July 2015. 
Introducción: El diagnóstico de un trombo intracardíaco tiene implicancias terapéuticas y pronósticas relevantes en el estudio y manejo del accidente cerebrovascular oclusivo ${ }^{1}$. En Chile la pesquisa diagnóstica de una probable fuente embolígena intracardíaca se realiza mediante imagen no invasiva ${ }^{2}$. En la práctica clínica diaria, la ecocardiografía es ampliamente aceptada como el test de tamizaje primario para la detección de un trombo intracardíaco $^{3}$. Esta práctica está sustentada por múltiples estudios los cuales demostraron que la ecocardiografía tiene una buena aproximación diagnóstica cuando el propósito del examen ecocardiográfico es la pesquisa de un trombo intraventricular ${ }^{4-5}$. Posteriormente con la aparición y uso del contraste ecocardiográfico el diagnóstico de trombo intraventricular mediante el uso del ultrasonido mejoró ostensiblemente ${ }^{6-7}$.

La Resonancia Magnética Cardíaca se ha desarrollado de manera progresiva durante las últimas 3 décadas. Actualmente corresponde a una tecnología única que puede ser usada para evaluar la función y morfología ventricular, perfusión, viabilidad y metabolismo cardíaco ${ }^{8}$. Hasta la fecha variados estudios han demostrado que esta tecnología es superior a las técnicas ecocardiográficas con el fin de pesquisar y diagnosticar la presencia de trombos intracardíacos ${ }^{9-12}$.

El objetivo de este estudio es describir los hallazgos de trombos intracardíacos en una serie de pacientes enviados a estudio de Resonancia Magnética Cardíaca, en los cuales el ecocardiograma transtorácico no estableció el diagnóstico de trombo.

\section{Métodos:}

Pacientes: Se realizó un análisis descriptivo de una serie de 62 casos con diagnóstico confirmado mediante Resonancia Magnética Cardíaca de un trombo intracardíaco, todos con ecocardiograma transtorácico previo realizado en un plazo no mayor de tres meses previos a la resonancia y sin trombos visibles. Estos pacientes fueron enviados a resonancia cardíaca por estudio cardiovascular el cual, en su gran mayoría, no contemplaba un estudio de pesquisa de fuente embolígena: Estudio de cardiomiopatía no isquémica en el $41 \%$ de los casos; viabilidad en el $41 \%$ $\mathrm{y}$ accidente vascular encefálico isquémico sin fuente embolígena demostrada por otros medios en el $18 \%$ restante. Los exámenes fueron realizados en Vanderbilt University Medical Center, Nashville, Tennessee, Estados Unidos, entre Julio del 2014 a Julio del 2015. Los pacientes eran mayoritariamente hombres $(72 \%)$, con un rango de edad entre 18 y 84 años, con disfunción sistólica de VI severa el $63 \%$; moderada $26 \%$ y normal en el $11 \%$. El $38 \%$ de los
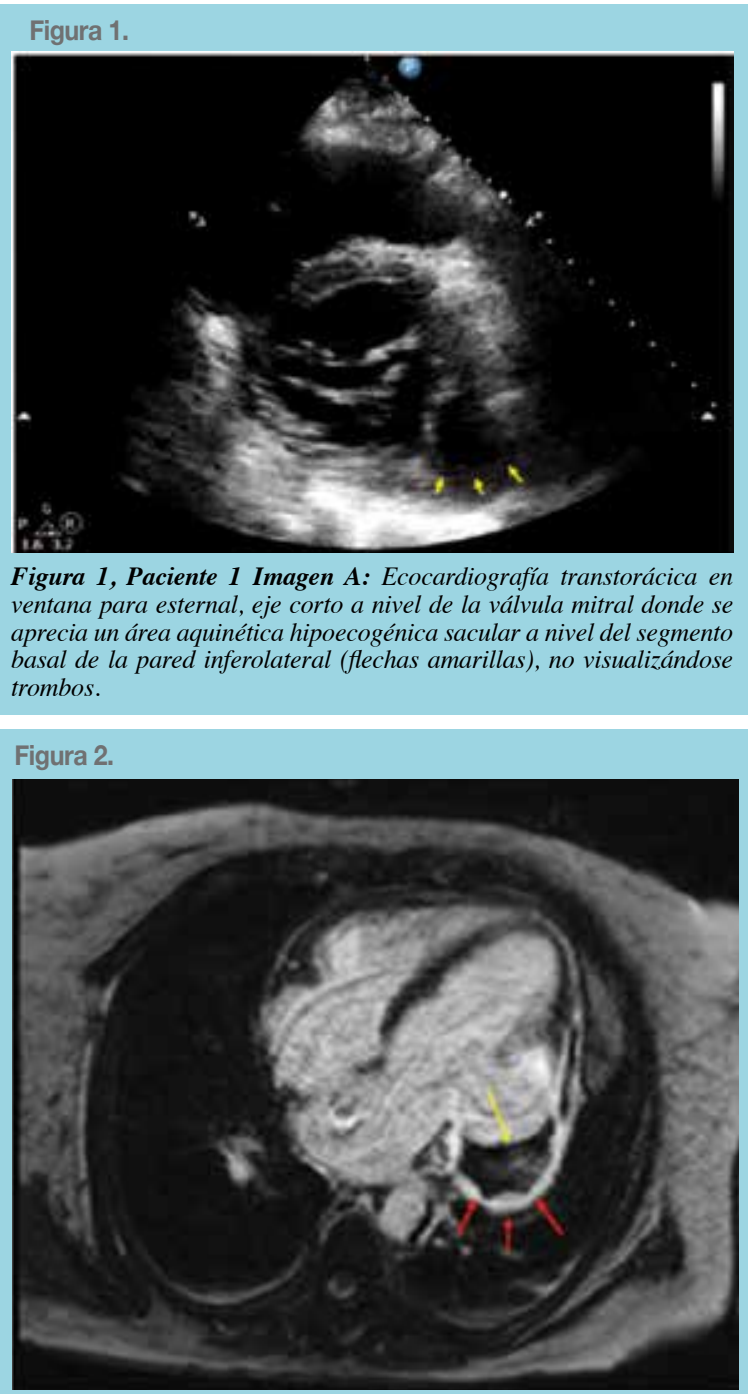

Figura 2, Paciente 1 Imagen B: Resonancia Magnética cardíaca secuencia "Late Gadolinium Enhancement - single shot inversión recovery FISP" en visión cuatro cámaras, en la que se aprecia un infarto antiguo transmural inferolateral con desarrollo de un pseudoaneurisma (flechas rojas). Dentro de esta cavidad se aprecia un trombo organizado (flecha amarilla).

sujetos presentaba historia presente o remota de Accidente vascular Encefálico.

Adquisición de las imágenes: El estudio por Resonancia Magnética Cardíaca se realizó usando un magneto de 1.5 Tesla (Siemens Avanto, Siemens, Malvern, Pennsylvania, Estados Unidos). El protocolo de Resonancia Magnética Cardíaca consistió en tres componentes: Cine Resonancia Magnética Cardíaca para la evaluación anatómica y funcional, Realce temprano de Gadolinio y Realce tardío de Gadolinio. La Cine Resonancia Magnética Cardíaca utilizó un protocolo true-FISP (Tiempo de repetición de $3 \mathrm{~ms}$, tiempo de Eco de $1.5 \mathrm{~ms}$, resolución espacial 1.7 x $1.4 \mathrm{~mm}$, resolución temporal de 35 a 40 ms). El protocolo de Re- 

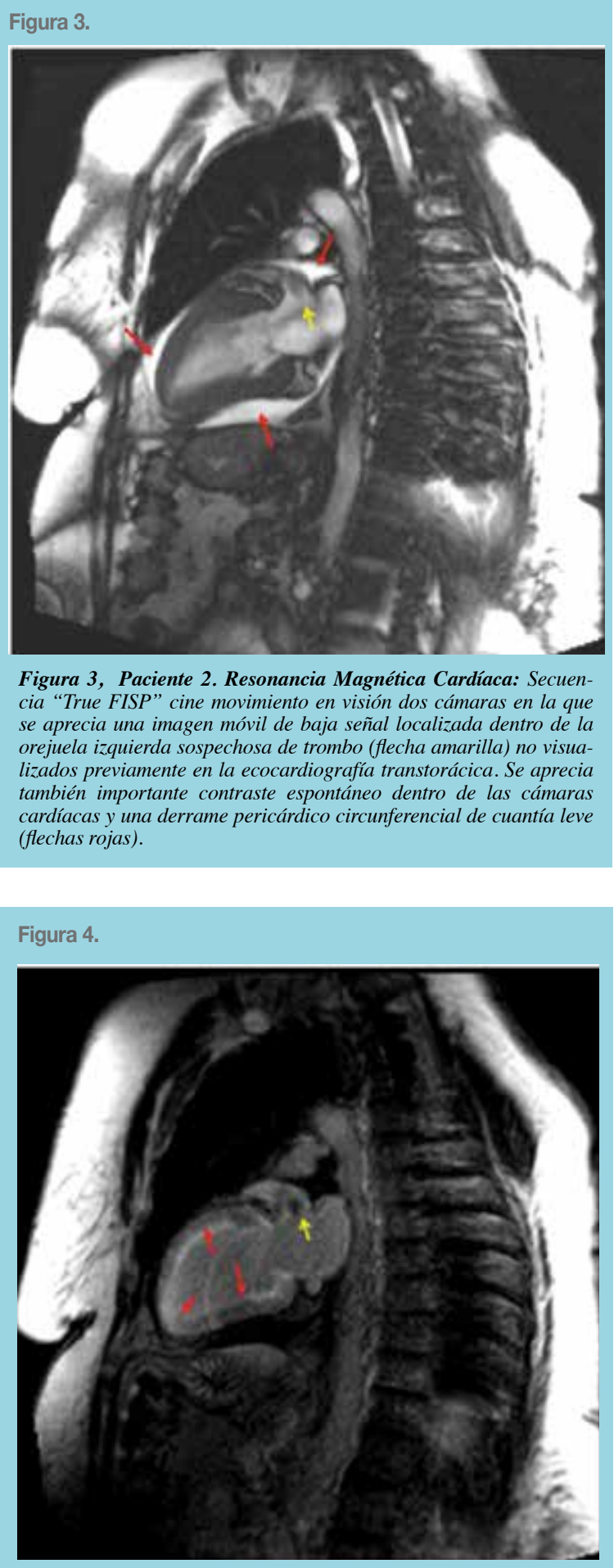

Figura 4, Paciente 2. Resonancia Magnética Cardíaca: Secuencia "Late Gadolinium Enhancement inversión recovery FISP" en visión dos cámaras en la que se aprecia la misma imagen señalada en la foto anterior localizada dentro de la orejuela izquierda la cual es avascular compatible con trombo (flecha amarilla) no visualizado previamente en la ecocardiografía transtorácica. Se aprecia también importante realce de Gadolinio de todo el miocardio incluido la pared auricular izquierda con disposición desde el endocardio al epicardio (flechas rojas) compatible con amiloidosis cardíaca. alce temprano de Gadolinio se realizó entre 1 a 3 minutos posterior a la administración de Gadolinio dependiendo del tipo de preparado farmacéutico de acuerdo al clearance de creatinina con varias secuencias true-FISP de disparo único, con tiempo de inversión de $600 \mathrm{~ms}$ en un plano cardíaco en (resolución espacial de 1.7 x $1.4 \mathrm{~mm}$ ). En casos de clearance de creatinina de $30 \mathrm{~mL} / \mathrm{min}$ o más se utilizó Gadopentetato de Dimeglumina (Magnevist ${ }^{\circ}$, Bayer HealthCare Pharmaceuticals Inc, Berlin, Alemania) en dosis de $0.2 \mathrm{~mL} / \mathrm{kg}(0.1$ $\mathrm{mmol} / \mathrm{kg}$ ). En los casos de pacientes que presentaban un clearance de creatinina entre 20 y $29 \mathrm{~mL} / \mathrm{min}$ se utilizó Gadobutrol (Gadavist ${ }^{\circledR}$, Bayer HealthCare Pharmaceuticals Inc, Berlin, Alemania) en dosis de $0.1 \mathrm{~mL} / \mathrm{kg}(0.1 \mathrm{mmol} /$ $\mathrm{kg}$ ). En este protocolo los trombos típicamente se visualizan de un color gris-negro y el miocardio normalmente de un color gris-claro. El protocolo de Realce Tardío de Gadolinio se obtuvo 10 minutos posterior a la administración de Gadolinio, utilizándose varias secuencias de tiempo de recuperación FISP de disparo único y tiempo de recuperación segmentado usualmente entre 250 a $300 \mathrm{~ms}$ en distintos planos cardíacos, usualmente eje corto y ejes largos dos cámaras, eje largo de cuatro cámaras y eje largo en visión de tracto de salida del ventrículo izquierdo. En este último protocolo los trombos típicamente tienen un color gris en su centro y con bordes negros, el miocardio tiene color negro y el tejido miocárdico infartado tiene color blanco.

Ecocardiografía: La ecocardiografía transtorácica fue realizado por sonografistas formalmente entrenados y con experiencia en equipos comercialmente disponibles (iE33 xMATRIX o CX50 CompactXtreme, Philips Healthcare, Andover, Massachusetts, Estados Unidos) usando transductores de matriz de fase. Las imágenes fueron adquiridas en las visiones estandarizadas paraesternal eje largo y eje corto, ventana apical en visión cuatro, tres y dos cámaras, ventana subcostal y ventana supraesternal. Todos los estudios ecocardiográficos fueron realizados como parte de la práctica clínica rutinaria. También se utilizó Perflutren lipídico (Definity®, Lantheus Medical Imaging, North Billerica, Massachusetts, Estados Unidos) en los casos en los cuales por criterio del sonografista fue necesario ya sea para opacificar la cavidad ventricular o delinear los bordes internos del endocardio. Las imágenes fueron almacenadas digitalmente, posteriormente revisadas y analizadas utilizando una estación de trabajo "HeartLab" (Agfa).

Análisis de datos: Las imágenes obtenidas mediante ecocardiografía fueron analizadas de manera aleatoria por ocho cardiólogos con experiencia y entrenamiento formal 


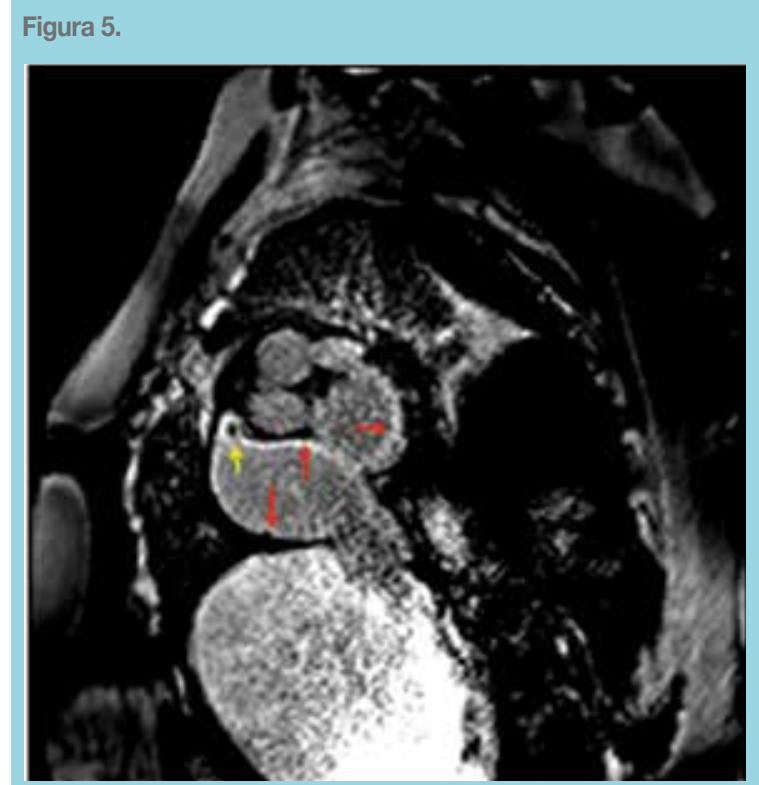

Figura 5, Paciente 2. Resonancia Magnética Cardíaca: Secuencia "Late Gadolinium Enhancement inversión recovery FISP" en visión eje corto a nivel bi-auricular en la que se aprecia una imagen avascular localizada en el vértice superior de la orejuela derecha compatible con trombo (flecha amarilla) no visualizado previamente en la ecocardiografía transtorácica. Se aprecia también importante realce de Gadolinio de toda la pared auricular derecha e izquierda con disposición desde el endocardio al epicardio compatible con amiloidosis cardiaca (flechas rojas).

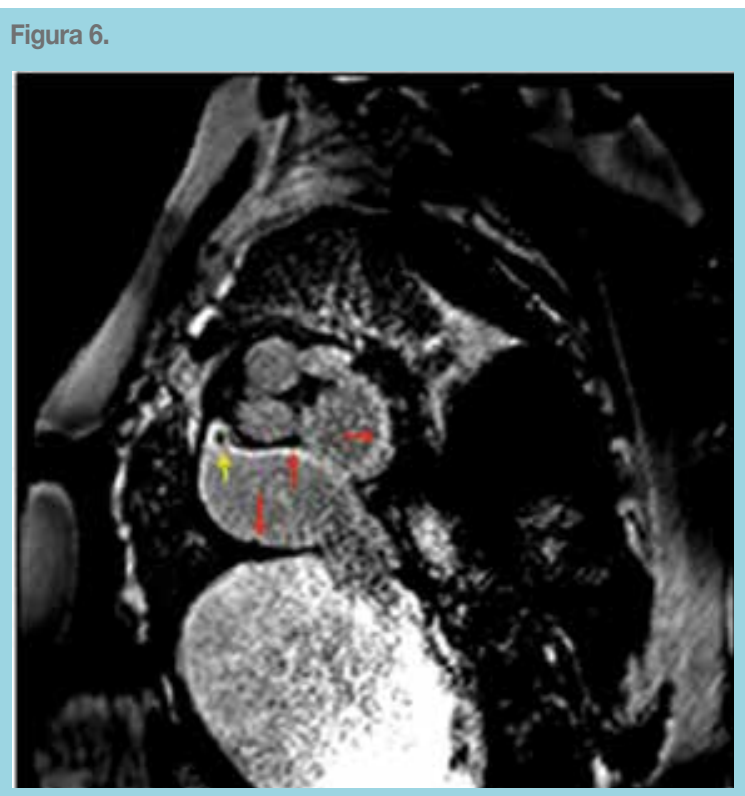

Figura 6, Paciente 3. Resonancia Magnética Cardíaca: Secuencia "Early Gadolinium Enhancement Time Inversion 600 ms" en visión dos cámaras en la que se aprecia una imagen avascular localizada en el aspecto endocárdico de la pared apical compatible con trombo (flecha amarilla) no visualizado previamente en la ecocardiografía transtorácica. Se aprecia también un importante adelgazamiento del miocardio localizado en la pared apical del segmento medio y distal de la pared anterior y del segmento distal de la pared inferior, sugerente de un infarto antiguo no transmural versus una isquemia crónica de dichas paredes (flechas rojas). en ecocardiografía (Niveles COCATS II y III) quienes tenían acceso a la historia clínica del paciente durante la interpretación del examen. Las imágenes obtenidas mediante Resonancia Magnética Cardíaca fueron analizadas de manera aleatoria por tres cardiólogos con experiencia y entrenamiento formal en Resonancia Magnética Cardíaca (COCATS nivel III) quienes tenían acceso a la historia clínica durante el examen. Para el diagnóstico de trombo mediante Resonancia Magnética Cardíaca se utilizó el criterio de imagen avascular visualizada en protocolo FISP y realce temprano de Gadolinio con tiempo de inversión de $600 \mathrm{~ms}$ en el cual posteriormente se confirmaba con protocolo ya sea de disparo único FISP o segmentado asociado a realce tardío de Gadolinio. De este modo se visualiza el trombo como una estructura avascular de baja intensidad rodeado por una estructura de alta intensidad.

Una vez identificado el trombo, este fue catalogado como Pediculado si el diámetro de la base o sitio de implantación de este era menor que el diámetro mayor localizado en su extremo libre, Cécil si el diámetro de la base o sitio de implantación era igual o mayor que el diámetro mayor localizado en su extremo libre, o Laminar si las paredes del trombo eran contiguas a la pared cardíaca a la cual estaba adherida y no presentaba protuberancias dentro de la cavidad cardíaca. La localización del trombo fue catalogado de acuerdo a su ubicación en las cavidades cardíacas: ventrículo izquierdo, ventrículo derecho, aurícula izquierda (sin incluir la orejuela izquierda), aurícula derecha (sin incluir la orejuela derecha), orejuela izquierda y orejuela derecha. Los datos fueron expresados en tablas y las variables comparadas en porcentajes.

\section{Resultados:}

Las imágenes correspondientes a trombos cardíacos fueron localizados en ventrículo izquierdo en su gran mayoría (58 casos, $94 \%)$, orejuela de aurícula izquierda $(3,2 \%)$ y ambas orejuelas (1 caso, $0.6 \%)$. Los trombos fueron en su gran mayoría laminares ( 52 casos, $84 \%$ ), sésiles ( 10 casos, $16 \%$ ) y ninguno pediculado. Las Figuras 1 a 8 , ilustran los hallazgos morfológicos.

\section{Discusión:}

La ecocardiografía transtorácica corresponde a la modalidad de examen de imagen cardíaca más utilizada en Chile. Normalmente dentro de la práctica clínica nacional se le ha otorgado una posición preponderante frente a la sospecha de una fuente embolígena cardíaca dejando a la ecocardiografía transesofágica como método de evaluación en el contexto de la fibrilación auricular, la sospecha de un trom- 

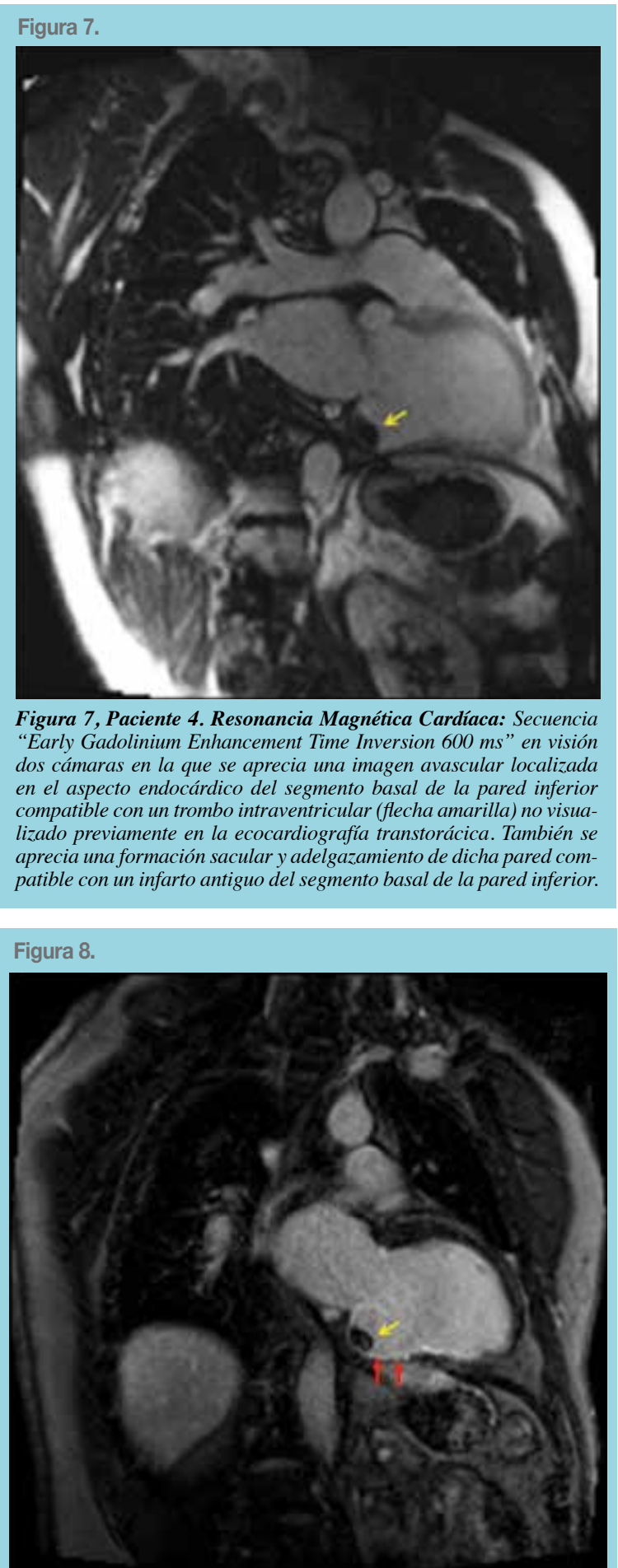

Figura 8, Paciente 4. Resonancia Magnética Cardíaca: Secuencia "Late Gadolinium Enhancemente Inversion recovery FISP" en visión dos cámaras en la que se aprecia una imagen avascular localizada en el aspecto endocárdico del segmento basal de la pared inferior compatible con un trombo intraventricular (flecha amarilla) no visualizado previamente en la ecocardiografía transtorácica. También se aprecia una formación sacular y adelgazamiento de dicha pared asociado a realce tardío de Gadolinio de predominio endocárdico que se extiende hasta el segmento medio de la pared inferior, hallazgos compatibles con un infarto antiguo del segmento basal y medio de la pared inferior (flechas rojas). bo localizado dentro de la orejuela izquierda o sospecha de un trombo intraauricular. Es sabido que la ecocardiografía transtorácica indicada por evaluación cardiovascular estándar tiene una sensibilidad de $33 \%$ y una especificidad de 91\% ${ }^{12}$ comparado con Resonancia Magnética Cardíaca para el diagnóstico de trombo intracardíaco. Asimismo, dentro de la práctica clínica el estudio ecocardiografíco transesofágico ha demostrado un incremento de su rendimiento diagnóstico cuando la indicación es la sospecha de trombo intracardíaco, alcanzando una sensibilidad de un $60 \%$ pero con una especificidad de un $88 \%^{12}$ comparado con la Resonancia Magnética Cardíaca. Dada la evidencia ya establecida y, tomando como ejemplo los casos en el presente estudio, se puede cuestionar el alcance real del examen ecocardiográfico transtorácico como método de pesquisa, diagnóstico y exclusión real de un trombo intracardíaco, tanto en pacientes que padecen un accidente cerebrovascular isquémico como aquellos que presentan un ataque isquémico transitorio. Por otro lado, el uso de la Resonancia Magnética Cardíaca en distintos escenarios clínicos cardiovasculares, ha demostrado la presencia de trombos intracardíacos que no se sospechaban y que eventualmente no fueron diagnosticados mediante el examen transtorácico estándar.

Debemos señalar que la verdadera comparación del rendimiento de la Ecocardiografía transtorácica y la RNM para el diagnóstico de trombos hubiera requerido efectuar esta última en todos los casos estudiados con ecografía, referidos por los diagnósticos ya señalados. Naturalmente, esta opción requeriría un estudio prospectivo con consentimiento de los pacientes y aprobación específica por el Comité de Ética institucional.

Actualmente, las guías chilenas de manejo del accidente cerebrovascular isquémico en personas mayores de 15 años $^{2}$ establecen como recomendación un "estudio vascular y cardíaco" para determinar la etiopatogenia de un ataque isquémico transitorio con un nivel de evidencia $\mathrm{C}$ no especificando el tipo de estudio cardíaco. La evidencia descrita en este estudio y la descrita en la literatura, invitan a considerar la Resonancia Magnética Cardíaca como el examen de imagen preferente tanto para pesquisar, diagnosticar y excluir la presencia de trombos intracardíacos en nuestro medio. Es conveniente tener en cuenta que para el diagnóstico de trombos pequeños localizados en la orejuela izquierda el Eco transesofágico pueda ser más confiable que la RNM. Finalmente, debiera crearse un Score de riesgo de trombogenicidad cardíaca para identificar los pacientes con enfermedad cardiovascular que ameritan un estudio por Resonancia Magnética para el diagnóstico de trombos intracardíacos en general. 


\section{Referencias:}

1. KERNAN W, OVBIAGELE B, CHARI V, BLACK H, BRAVATA D, CHIMOWITZ M, et al; on behalf of the American Heart Association Stroke Council, Council on Cardiovascular and Stroke Nursing, Council on Clinical Cardiology, and Council on Peripheral Vascular Disease. AHA/ASA Guidelines for the Prevention of Stroke in Patients With Stroke and Transient Ischemic Attack. Stroke 2014; 45: 2160-2236.

2. Serie Guías Clínicas MINSAL 2013. Accidente Cerebro Vascular Isquémico en personas de 15 años y más. Guía Clínica AUGE. Subsecretaría de Salud Pública. División de Prevención y Control de Enfermedades Secretaria Técnica AUGE. web.minsal.cl

3. ACCF/ASE/AHA/ASNC/HFSA/HRS/SCAI/SCCM/SCCT/ SCMR 2011 Appropriate Use Criteria for Echocardiography. Journal of the American Society of Echocardiography, Volume 24, Issue 3, 229-267.

4. VISSER CA, KAN G, DAVID GK, LIE KI, DURRER D. Two dimensional echocardiography in the diagnosis of left ventricular thrombus. A prospective study of 67 patients with anatomic validation. Chest 1983; 83: 228-232.

5. STRATTON JR, LIGHTY GW JR., PEARLMAN AS, RITCHIE JL. Detection of left ventricular thrombus by two-dimensional echocardiography: sensitivity, specificity, and causes of uncertainty. Circulation 1982; 66: 156-166.

6. THANIGARAJ S. SCHECHTMAN KB, PEREZ JE. Improved echocardiographic delineation of left ventricular thrombus with the use of intravenous second generation contrast image enhan- cement. J Am Soc Echocardiogr 1999; 12: 1022-1026.

7. MANSENCAL N, NASR IA, PILLIÈRE R, FARCOT JC, JOSEPH T, LACOMBE P, et al. Usefulnes of contrast echocardiography for assessment of left ventricular thrombus after acute myocardial infarction. Am J Cardiol 2007; 99: 1667-1670.

8. POHOST G. The History of Cardiovascular Magnetic Resonance. J Am Coll Cardiol Img. 2008; 1: 672-678.

9. BARKHAUSEN J, HUNOLD P, EGGEBRECHT H, et al. Detection and characterization of intracardiac thrombi on MR imaging. AJR Am J Roentgenol 2002;179:1539-1544.

10. SRICHAI MB, JUNOR C, RODRIGUEZ LL, et al. Clinical, imaging, and pathologic characteristics of left ventricular thrombus: a comparison of contrast enhanced magnetic resonance imaging, transthoracic echocardiography and transesophageal echocardiography with surgical or pathological validation. Am Heart J 2006;152:75-84.

11. WEINSAFT J, KIM H, SHAH DJ, et al. Detection of left ventricular thrombus by delayed-enhancement CMR: prevalence and markers in patients with systolic dysfunction. J Am Coll Cardiol 2008;52:148-157.

12. WEINSAFT JW, KIM HW, CROWLEY AL, KLEM I, SHENOY C, VAN ASSCHE L, BROSNAN R, SHAH DJ, VELASQUEZ EJ, PARKER M, JUDD RM, KIM RJ. LV thrombus detection by routine echocardiography: insights into performance characteristics using delayed enhancement CMR. JACC Cardiovasc Imaging. 2011 Jul;4(7):702-712. 